\title{
Definition of Nonresponse to Analgesic Treatment of Arthritic Pain: An Analytical Literature Review of the Smallest Detectable Difference, the Minimal Detectable Change, and the Minimal Clinically Important Difference on the Pain Visual Analog Scale
}

\author{
Melissa E. Stauffer, ${ }^{1}$ Stephanie D. Taylor, ${ }^{2}$ Douglas J. Watson, ${ }^{2}$ \\ Paul M. Peloso, ${ }^{2}$ and Alan Morrison ${ }^{1}$ \\ ${ }^{1}$ Scribco Pharmaceutical Writing, P.O. Box 1525, Blue Bell, PA 19422, USA \\ ${ }^{2}$ Departments of Global Health Outcomes, Epidemiology, and Clinical Development, Merck \& Co., Inc., \\ One Merck Drive, Whitehouse Station, NJ 08889, USA \\ Correspondence should be addressed to Melissa E. Stauffer, melstauff@charter.net
}

Received 25 January 2011; Accepted 8 March 2011

Academic Editor: Bernhard Rintelen

Copyright () 2011 Melissa E. Stauffer et al. This is an open access article distributed under the Creative Commons Attribution License, which permits unrestricted use, distribution, and reproduction in any medium, provided the original work is properly cited.

Our objective was to develop a working definition of nonresponse to analgesic treatment of arthritis, focusing on the measurement of pain on the $0-100 \mathrm{~mm}$ pain visual analog scale (VAS). We reviewed the literature to assess the smallest detectable difference (SDD), the minimal detectable change (MDC), and the minimal clinically important difference (MCID). The SDD for improvement reported in three studies of rheumatoid arthritis was 18.6, 19.0, and 20.0. The median MDC was 25.4 for 7 studies of osteoarthritis and 5 studies of rheumatoid arthritis (calculated for a reliability coefficient of 0.85 ). The MCID increased with increasing baseline pain score. For baseline VAS tertiles defined by scores of 30-49, 50-65, and >65, the MCID for improvement was, respectively, 7-11 units, 19-27 units, and 29-37 units. Nonresponse can thus be defined in terms of the MDC for low baseline pain scores and in terms of the MCID for high baseline scores.

\section{Introduction}

Nonsteroidal anti-inflammatory drugs (NSAIDs) are the first-line treatment for osteoarthritis [1] and a cornerstone of pharmacologic management of other arthritic and rheumatologic illnesses [2]. The dichotomous classification of patients into responders and nonresponders to NSAIDs began in the 1970s [3]. Walker et al. provided evidence of the validity of the responder/nonresponder thesis in rheumatoid arthritis and osteoarthritis [4].

Both the American College of Rheumatology (ACR) and the European League Against Rheumatism (EULAR) have developed response criteria for rheumatoid arthritis. The ACR criteria are based on a core set of measures with 7 components [5]. EULAR response criteria are based on the multiitem Disease Activity Score 28 (DAS28) [6]. A composite index of patient-reported pain, physical function, and global assessment appears to be as good as the entire ACR core set or the DAS28 for determining response to treatment in clinical trials of rheumatoid arthritis [7].

The most recent definition of response to NSAID treatment in osteoarthritis was determined by a joint task force comprising members of the Outcome Measures in Rheumatology (OMERACT) committee and the Osteoarthritis Research Society International (OARSI) [8]. In the OMERACT-OARSI guidelines, response is a binary, composite endpoint based on three patient-reported core outcome measures: pain, physical function, and the patient global assessment. Of these three, pain is considered the primary outcome measure of interest [9].

In clinical trials, definitions of the effectiveness of analgesic therapies for arthritic pain are focused on response to treatment. In clinical practice, however, decisions to change the analgesic dose or drug for an individual patient 
are based on nonresponse to the existing treatment. We sought to develop a working definition of nonresponse to analgesic treatment, focusing on the measurement of pain with the $0-100 \mathrm{~mm}$ visual analog scale (VAS). We posited that nonresponse could be measured in terms of (i) the smallest amount of change on the pain VAS that can be reliably measured or (ii) the smallest change that the patients report as important. The first of these concepts, the smallest amount of change that can be reliably measured, is reported in the literature as either the smallest detectable difference (SDD) or the minimal detectable change (MDC). The second concept is reported as the minimal clinically important difference (MCID).

\section{Literature Search}

Searches were carried out for randomized clinical trials (RCTs) of patient-reported measurement of chronic pain in arthritis. Articles known to the authors were supplemented by searches of PubMed performed in June 2010 with the following algorithms: reliability AND pain AND VAS; *arthritis AND "Drug Resistance" [MeSH Terms] AND "anti-inflammatory agents, Nonsteroidal" [MeSH Terms] AND "humans" [MeSH Terms]; NSAID-refractory; NSAID* AND (non-respon* OR nonrespon*); refractory [title word] AND osteoarthritis [title word]; refractoriness [title word] AND concept. These searches resulted in 318 unique items. Articles were screened for those reporting parameters of the English $0-100 \mathrm{~mm}$ pain VAS: the SDD, the MCID, or the reliability, from which the MDC can be calculated.

\section{The Smallest Detectable Difference}

The SDD on a measurement scale is that amount of difference for which anything smaller cannot be reliably distinguished from random error in the measurement [12]. There are two methods of estimating this quantity in original scale units [12]. The first is the limits of agreement method [13], and the term SDD is usually used when the parameter is calculated by this method. The SDD is applicable to longitudinal data [14], and thus the limits of agreement method is often used to assess agreement between test and retest scores $[12,13]$. The mean difference between test and retest scores and its SD are calculated, and the SDD is the $95 \%$ confidence interval (CI) of the difference, that is, 1.96 times the SD of the difference between scores [11].

The SDD reported in three RCTs of rheumatoid arthritis, given as negative change or improvement, was $-18.6,-19.0$, and -20.0 (Table 1). SDDs of this magnitude constitute half or more of the mean baseline pain score in VAS units, suggesting that a very large change in pain status is required before that change is detectable.

\section{Minimal Detectable Change}

The second method for calculating the SDD is from the standard error of the measurement (SEM) $[15,16]$, and, when calculated in this way, it is usually termed the MDC (it is also referred to as the "smallest real difference" [17] and the "tolerance interval" [18]). The SEM is a function of the standard deviation (SD) of the study sample and the reliability of the measurement instrument [19]. The reliability coefficient (RC) may be the test-retest reliability or an intraclass correlation coefficient. The MDC is thus applicable to cross-sectional data [14]. The MDC is given by MDC $= \pm 1.96 \times \sqrt{2} \times$ SEM where 1.96 derives from the $95 \%$ CI. The SEM is derived from the SD and the RC as follows: $\mathrm{SEM}=\mathrm{SD} \times \sqrt{(1-\mathrm{RC})}$.

The MDC was rarely reported in published RCTs of rheumatoid arthritis or osteoarthritis. Hence, we calculated it for RCTs reporting mean (SD) values from the pain VAS. We searched for RC values in the literature. Six RCTs of rheumatoid arthritis patients (the three in Table 1 and three additional publications) reported RCs of 0.74 [20], 0.75 [12], 0.88 [21], 0.88 [10], 0.94 [11], and 0.94 [22]. The median RC was 0.88 . We calculated the MDC for rheumatoid arthritis $[10,11,20,21,23,24]$ and osteoarthritis [25-30] RCTs reporting mean pain VAS score at baseline using three arbitrary RC values $(0.75,0.85$, and 0.95$)$ spanning the range found in the literature.

As expected based on the above formulas, the MDC was smaller (i.e., a more precise measurement could be obtained) for higher values of RC (Figure 1). For example, in osteoarthritis, the median MDCs were 10.7, 18.6, and 24.0 for RCs of $0.95,0.85$, and 0.75 , respectively. In addition, the data appear to indicate a trend toward smaller MDCs at higher baseline pain scores (Figure 1). By linear regression analysis, the slope of the line through all the data points (osteoarthritis and rheumatoid arthritis) with $\mathrm{RC}=0.85$ $(-0.28)$ was statistically significant at $P=0.005$ (as were the slopes at the other two RC values). This finding suggests that, when the patient's pain is greater, the instrument can reliably detect smaller differences in pain.

For a given RC, the MDC was larger for rheumatoid arthritis than for osteoarthritis (Figure 1). (The median MDCs for rheumatoid arthritis were 15.8, 27.3, and 35.3 for RCs of $0.95,0.85$, and 0.75 , resp.) Linear regression analyses of rheumatoid arthritis and osteoarthritis trials as separate groups did not produce statistically significant correlations between baseline pain and MDC. Rather than MDC being inversely correlated with baseline pain score, the data may be interpreted as a difference between osteoarthritis and rheumatoid arthritis, combined with the fact that the rheumatoid arthritis trials assessed here included patients with lower baseline pain scores than the patients in the osteoarthritis trials.

\section{Minimal Clinically Important Difference}

Distinct from the concepts of SDD and MDC is that of the minimal clinically important difference (MCID). Jaeschke et al. defined the MCID as "the smallest difference in score in the domain of interest which patients perceive as beneficial and which would mandate...a change in the patient's management" [31]. 
TABLE 1: Smallest detectable difference (SDD) of the 0-100 mm pain VAS reported in studies of rheumatoid arthritis.

\begin{tabular}{lccccc}
\hline \multirow{2}{*}{ Study } & \multirow{2}{*}{$N$} & \multicolumn{2}{c}{ Baseline pain VAS score } & \multicolumn{2}{c}{ SDD } \\
& & Mean & SD & Absolute value & As \% of pain VAS score \\
\hline Uhlig et al. 2009 [10] & 28 & 29.5 & 23.7 & $-18.6,+26.0$ & $\pm 76 \%$ \\
Pincus et al. 2008 [11] & 264 & $39.8^{\mathrm{c}}$ & $29.1^{\mathrm{c}}$ & $-19.0,+19.4$ & $\pm 48 \%$ \\
Lassere et al. 2001 [12] & 24 & 37 & - & $-20,+16.3$ & $\pm 49 \%$ \\
\hline
\end{tabular}

SD: standard deviation; SDD: smallest detectable difference; VAS: visual analog scale.

${ }^{\mathrm{a}} 0-100 \mathrm{~mm}$ pain VAS from 0 , no pain, to 100 , very severe pain.

b All 10-mm scale values were converted to a 100-point scale by multiplying by ten.

"Values provided by T. Pincus and C. Swearingen for the "line 1" scores (see original article).

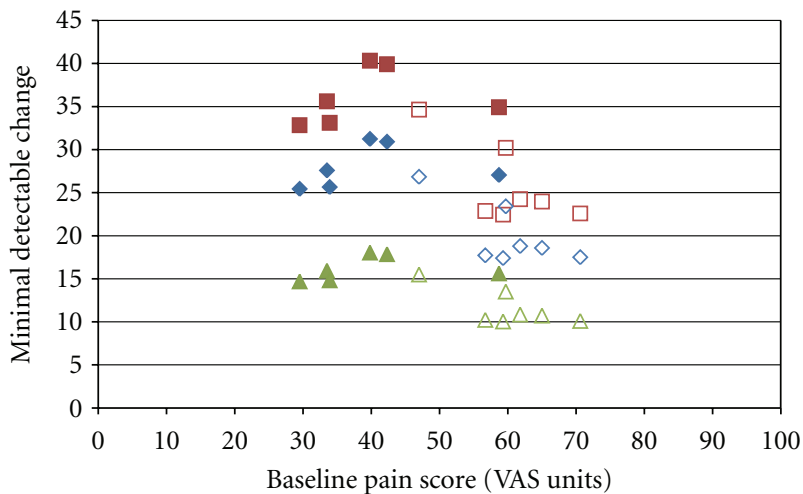

Figure 1: Minimal detectable change by baseline pain score, for different values of the reliability coefficient. Closed symbols show data for rheumatoid arthritis, while open symbols depict osteoarthritis. Squares show MDC at $\mathrm{RC}=0.75$, diamonds at $\mathrm{RC}$ $=0.85$, and triangles at $\mathrm{RC}=0.95$.

The measurement of the MCID for a scale requires a reference standard (or "external criterion"; also referred to as an "anchor" [32]) —frequently the patient global assessment (PGA) of treatment effect. The four RCTs reporting the MCID on the pain VAS used the PGA as an anchor (Table 2). Although the MCID values for improvement reported in these RCTs fall within a relatively narrow range $(-11.1$ to -19.9 ), there was no consensus on the definition of the MCID (Table 2). For the purposes of this paper, the MCID is expressed as the amount of change on the pain VAS corresponding to a specific degree of change on the PGA.

Tubach et al. [30] assessed only the improvement aspect of the MCID and defined the minimal clinically important improvement (MCII) as the minimum improvement (on the pain VAS) reported by $75 \%$ of osteoarthritis patients who ranked their response as "good" on the PGA. By this definition, the MCII was -15.3 for hip osteoarthritis and -19.9 for knee osteoarthritis (Table 2). The MCID for osteoarthritis pain was also investigated in terms of the "minimal perceptible clinical improvement" (MPCI) [26]. This parameter was defined as the change in the pain VAS score corresponding to a change from "none" to "poor" in the PGA, and the reported value was -11.1. To achieve a direct comparison between the MPCI and the MCII described above, we examined the 75th percentile of patients with a PGA of "good" in Ehrich et al. [26] and found that they had a minimum change of approximately -33 on the pain VAS, a much larger change than that reported by Tubach et al. [30].

The MCID on the pain VAS reported by Pope et al. for rheumatoid arthritis was similar to that for osteoarthritis (Table 2) [24]. Pope et al. anchored the pain score in a patient-reported overall assessment of disease status [24]. Their study and another on back pain [18] assessed the MCID for both improvement and worsening, and both RCTs found that the MCID was larger for improvement than for worsening: -11.8 versus +6.8 in the RA study and -18 versus +8 in the back pain study (Table 2 ).

The MCID on the pain VAS is not a fixed value; it depends to some extent on the baseline pain score [33]. To investigate this idea, Tubach et al. divided their study population into tertiles based on baseline pain scores [30]. When knee osteoarthritis patients were categorized according to their baseline pain scores, the MCIIs were 11, 27, and 37 units for the lowest, middle, and highest tertiles, respectively. For hip osteoarthritis patients, these values were 7, 24, and 30. These findings are depicted in Figure 2.

The study of back pain also reported MCIDs according to baseline pain score [18]. In this study, baseline pain scores were divided into three categories: mean minus $2 * S D$, mean, and mean plus $2 * \mathrm{SD}$, corresponding to scores of 35,64 , and 93, respectively. These scores fit the tertiles described by Tubach et al. As shown in Figure 2, the MCIDs for improvement in each tertile were 9, 19, and 29, respectively. The MCID of -11.9 reported by Pope et al. [24] at a mean baseline score of 42.3 also fits in well with the data in Figure 2.

\section{Discussion}

Two of the SDDs reported in the literature $(-18.6$ and -19.0 ; Table 1) fit well onto the graph of MDC values in Figure 1 (not shown). The corresponding RCs of 0.88 [10] and 0.94 [11], respectively, may explain why the SDDs fall squarely between the overall median values of MDC at $\mathrm{RC}=0.95$ (14.7) and RC $=0.85$ (25.4) on the graph. The third SDD $(-20)$ [12], which had an RC of 0.75 , did not fit the graph for that MDC data set. 
TABLE 2: Minimal clinically important difference on the 0-100 mm pain VAS.

\begin{tabular}{|c|c|c|c|c|c|c|c|}
\hline \multirow{2}{*}{ Study } & \multirow{2}{*}{ Patients } & \multicolumn{3}{|c|}{ Anchor } & \multicolumn{3}{|c|}{ MCID } \\
\hline & & Type & Points & Responses & Definition & $\begin{array}{c}\text { Points on } \\
\text { anchor scale }\end{array}$ & $\begin{array}{l}\text { Units on } \\
\text { VAS }^{\mathrm{a}}\end{array}$ \\
\hline $\begin{array}{l}\text { Pope et al. } \\
2009[24]\end{array}$ & RA & PGA & 5 & $\begin{array}{l}\text { Much worsened, somewhat } \\
\text { worsened, same, somewhat } \\
\text { improved, much improved }\end{array}$ & $\begin{array}{l}\text { Difference between "same" and } \\
\text { "somewhat improved or } \\
\text { somewhat worsened" }\end{array}$ & \pm 1 point & $-11.9,+6.8$ \\
\hline $\begin{array}{l}\text { Tubach et al. } \\
2005[30]\end{array}$ & OA & PGA & 5 & $\begin{array}{l}\text { None, poor, fair, good, } \\
\text { excellent }\end{array}$ & $\begin{array}{l}\text { Difference between "none" and } \\
\text { "good" response }\end{array}$ & +3 points & $\begin{array}{l}-19.9 \text { (knee) } \\
-15.3 \text { (hip) }\end{array}$ \\
\hline $\begin{array}{l}\text { Hägg et al. } \\
2003[18]\end{array}$ & Back pain & PGA & 4 & $\begin{array}{l}\text { Worse, unchanged, better, } \\
\text { much better }\end{array}$ & $\begin{array}{l}\text { Difference between "unchanged' } \\
\text { and "better" or "worse" }\end{array}$ & \pm 1 point & $-18,+8$ \\
\hline $\begin{array}{l}\text { Ehrich et al. } \\
2000[26]\end{array}$ & $\mathrm{OA}$ & PGA & 5 & $\begin{array}{l}\text { None, poor, fair, good, } \\
\text { excellent }\end{array}$ & $\begin{array}{l}\text { Difference between "none" and } \\
\text { "poor" response on the PGA }\end{array}$ & +1 point & -11.1 \\
\hline
\end{tabular}

MCID; minimal clinically important difference; PGA: patient global assessment of response.

${ }^{a}$ Negative values indicate improvement, and positive ones indicate deterioration.

${ }^{b}$ Defined as minimal perceptible clinical improvement.

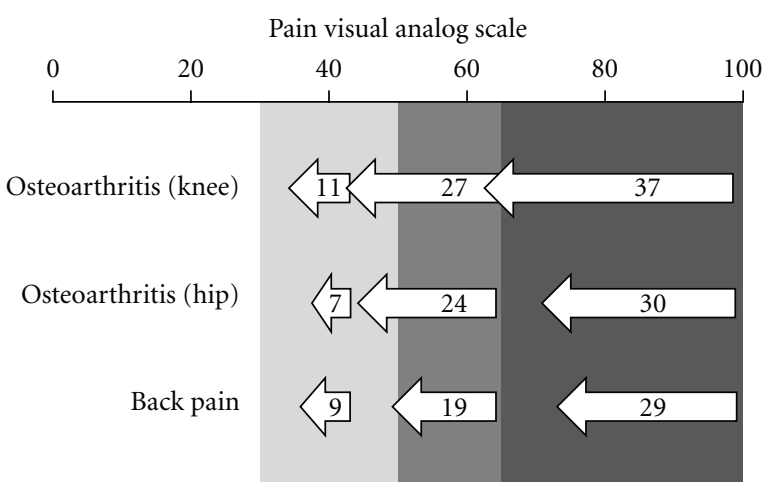

FIGURE 2: Minimal clinically important difference by tertile of baseline VAS score. The light, medium, and dark gray sections of the VAS $(30-49,50-65$, and $>65)$ correspond to the rounded tertiles of baseline pain VAS scores reported by Tubach et al. [30]. Arrows depict the MCID for improvement reported by Tubach et al. (osteoarthritis of knee and hip) [30] and Hägg et al. (back pain) [18].

The calculated values of MDC decreased with increasing baseline pain score, suggesting that small differences in pain can be measured more precisely at higher levels of pain. The MDC is a function of the SD of the mean grouplevel pain score. Is the SD for patient-reported pain actually smaller at higher levels of pain? According to these data, yes. Linear regression analyses of the SD versus the baseline pain score yielded a statistically significant slope $(-0.26$; $P=0.005)$ for the combined data set, but statistically nonsignificant slopes when osteoarthritis and rheumatoid arthritis were analyzed separately. There was also no linear relationship between the RC (the other variable on which MDC depends) and the baseline pain. We interpret this to mean that the relationship between the MDC and baseline pain is an inherent characteristic of the pain VAS rather than a byproduct of physiologically different disease states or the statistical reliability of the scale.
The trend of the MCID is opposite that of the MDCit is larger at higher baseline pain levels. Thus it appears that, although small differences in pain are reliably detectable in patients with severe pain, these patients require a larger amount of pain relief in order to report being satisfied with the change they experience. In the context of Figure 2, a patient with severe pain (score $>65$ ) needs a change to the moderate pain category (50-65) to view it as clinically important.

The relationship between MCID and baseline severity may apply to other measures in addition to the pain VAS. The RCTs included in our analysis often reported physical function and PGA as well and in Tubach et al. [30], for instance, the MCID for both the WOMAC physical function score and the PGA increased with increasing baseline scores. Future analyses should investigate the relationship between baseline pain and these other measures of patient response.

The OMERACT-OARSI criteria for response to treatment include both absolute and relative changes on the pain VAS. As described by Pham et al. [8], a responder experiences either (i) $\geq 50 \%$ improvement on the pain VAS, defined as at least 20 units, or (ii) $\geq 20 \%$ improvement, defined as at least 10 units, in combination with abovethreshold improvements on at least one other measure of patient response, that is, physical function or the PGA. The ACR criteria have a similar definition of response, graded by percentage improvements $(20 \%, 50 \%$, and $70 \%)$ in tender and swollen joint counts and 3 of the 5 other outcome measures (of which pain is one). However, the ACR20, ACR50, and ACR70 response levels are not linked to absolute unit improvements on the pain VAS. The question is whether the pain VAS is sensitive enough to detect the amount of change required for a definition of 'response' under either set of criteria.

At $\mathrm{RC}=0.85$, the median $\mathrm{MDC}$ on the pain VAS is 25.4 units (for osteoarthritis and rheumatoid arthritis combined), which is greater than the minimum of 1020 units required to be considered a responder in the OMERACT-OARSI criteria. As a percentage of the baseline 
pain, the median MDC is $46.1 \%$ (at $\mathrm{RC}=0.85$ ), which is within the threshold of improvement required for a response by definition (i) above, but outside the range required in definition (ii). This amount of change also meets the definition of an ACR20 response. Similarly, the SDDs in patients with RA, as shown in Table 1 (48\%, $49 \%$, and $76 \%$ ), indicate that the smallest detectable change in pain is greater than the minimum amount considered necessary for a definition of "response" by both sets of criteria.

The median values of MCID for each tertile in Figure 2 are 9, 24, and 30 units overall and 9, 25.5, and 33.5 units for osteoarthritis only. Whether the results are combined or osteoarthritis is considered separately, only patients attaining the MCID in the middle and highest tertiles can be classified as responders by the OMERACT-OARSI criteria. Those in the lowest tertile might perceive an improvement in their pain as clinically important but still be classified as a nonresponder. Taking the midpoint of each tertile in Figure 2 as the baseline pain score and the overall median value of MCID of each tertile as the true MCID, the relative values of MCID are $22.5 \%, 47.0 \%$, and $36.4 \%$ for baseline pain scores of 30-49, 50-65, and $>65$. Thus the MCID for each tertile is less than the amount required for a response by OMERACTOARSI definition (i) mentioned before.

\section{Conclusions}

We have shown that the smallest decrease in pain that can be reliably measured, the MDC, is smaller than the MCID for scores of $>65$ on the $0-100$ pain VAS, but not for scores of $<50$. Improvements in the middle of the pain VAS may or may not be measurable depending on the type of pain, the baseline score, and the RC of the pain VAS. We conclude that, for baseline pain scores above 65, nonresponse can be defined as any change on the pain VAS that is smaller than the MCID. For patients with scores below 65, a change less than the MDC can be considered nonresponse; however, improvements that are greater than the MDC but not considered clinically important by the patient may also justify a change in therapy.

\section{Acknowledgments}

The authors are grateful to Drs. T. Pincus and C. Swearingen for providing unpublished data and to Julia Vishnevetsky, $\mathrm{MPH}$, for medical writing assistance. The study was funded by Merck \& Co., Inc.

\section{References}

[1] K. D. Brandt, "Second-line drug therapy for osteoarthritis," Clinical Medicine, vol. 1, no. 2, pp. 110-112, 2001.

[2] C. O. Bingham III, "Development and clinical application of COX-2-selective inhibitors for the treatment of osteoarthritis and rheumatoid arthritis," Cleveland Clinic Journal of Medicine, vol. 69, no. 1, pp. SI5-SI12, 2002.
[3] H. A. Capell, B. Konetschnik, and R. C. Glass, "Antiinflammatory analgesic drug responders and non-responders: a clinicopharmacological study of flurbiprofen," British Journal of Clinical Pharmacology, vol. 4, no. 5, pp. 623-624, 1977.

[4] J. S. Walker, R. B. Sheather-Reid, J. J. Carmody, J. H. Vial, and R. O. Day, "Nonsteroidal antiinflammatory drugs in rheumatoid arthritis and osteoarthritis: support for the concept of "responders" and 'nonresponders," Arthritis and Rheumatism, vol. 40, no. 11, pp. 1944-1954, 1997.

[5] D. T. Felson, J. J. Anderson, M. Boers et al., "The American College of Rheumatology preliminary core set of disease activity measures for rheumatoid arthritis clinical trials," Arthritis and Rheumatism, vol. 36, no. 6, pp. 729-740, 1993.

[6] A. M. Van Gestel, M. L. L. Prevoo, M. A. Van 'T Hof, M. H. Van Rijswijk, L. B. A. Van De Putte, and P. L. C. M. Van Riel, "Development and validation of the European League Against Rheumatism response criteria for rheumatoid arthritis," Arthritis and Rheumatism, vol. 39, no. 1, pp. 34-40, 1996.

[7] T. Pincus, V. Strand, G. Koch et al., "An index of the three Core Data Set patient questionnaire measures distinguishes efficacy of active treatment from that of placebo as effectively as the American College of Rheumatology 20\% response criteria (ACR20) or the Disease Activity Score (DAS) in a rheumatoid arthritis clinical trial," Arthritis and Rheumatism, vol. 48, no. 3, pp. 625-630, 2003.

[8] T. Pham, D. V. Der Heijde, M. Lassere et al., "Outcome variables for osteoarthritis clinical trials: the OMERACTOARSI set of responder criteria," Journal of Rheumatology, vol. 30, no. 7, pp. 1648-1654, 2003.

[9] M. C. Hochberg, R. D. Altman, K. D. Brandt, and R. W. Moskowitz, "Design and conduct of clinical trials in osteoarthritis: preliminary recommendations from a Task Force of the Osteoarthritis Research Society," Journal of Rheumatology, vol. 24, no. 4, pp. 792-794, 1997.

[10] T. Uhlig, T. K. Kvien, and T. Pincus, "Test-retest reliability of disease activity core set measures and indices in rheumatoid arthritis," Annals of the Rheumatic Diseases, vol. 68, no. 6, pp. 972-975, 2009.

[11] T. Pincus, M. Bergman, T. Sokka, J. Roth, C. Swearingen, and Y. Yazici, "Visual analog scales in formats other than a 10 centimeter horizontal line to assess pain and other clinical data," Journal of Rheumatology, vol. 35, no. 8, pp. 1550-1558, 2008.

[12] M. N. D. Lassere, D. Van der Heijde, K. R. Johnson, M. Boers, and J. Edmonds, "Reliability of measures of disease activity and disease damage in rheumatoid arthritis: implications for smallest detectable difference, minimal clinically important difference, and analysis of treatment effects in randomized controlled trials," Journal of Rheumatology, vol. 28, no. 4, pp. 892-903, 2001.

[13] J. M. Bland and D. G. Altman, "Statistical methods for assessing agreement between two methods of clinical measurement," Lancet, vol. 1, no. 8476, pp. 307-310, 1986.

[14] C. A. McHorney and A. R. Tarlov, "Individual-patient monitoring in clinical practice: are available health status surveys adequate?" Quality of Life Research, vol. 4, no. 4, pp. 293-307, 1995.

[15] D. E. Beaton, C. Bombardier, J. N. Katz et al., "Looking for important change/differences in studies of responsiveness. OMERACT MCID Working Group. Outcome Measures in Rheumatology. Minimal Clinically Important Difference," Journal of Rheumatology, vol. 28, no. 2, pp. 400-405, 2001. 
[16] H. C. de Vet, C. B. Terwee, R. W. Ostelo, H. Beckerman, D. L. Knol, and L. M. Bouter, "Minimal changes in health status questionnaires: distinction between minimally detectable change and minimally important change," Health and Quality of Life Outcomes, vol. 4, article 54, 2006.

[17] H. Beckerman, M. E. Roebroeck, G. J. Lankhorst, J. G. Becher, P. D. Bezemer, and A. L. M. Verbeek, "Smallest real difference, a link between reproducibility and responsiveness," Quality of Life Research, vol. 10, no. 7, pp. 571-578, 2001.

[18] O. Hägg, P. Fritzell, and A. Nordwall, “The clinical importance of changes in outcome scores after treatment for chronic low back pain," European Spine Journal, vol. 12, no. 1, pp. 12-20, 2003.

[19] K. W. Wyrwich, "Minimal important difference thresholds and the standard error of measurement: is there a connection?" Journal of Biopharmaceutical Statistics, vol. 14, no. 1, pp. 97-110, 2004

[20] G. Rohekar and J. Pope, “Test-retest reliability of patient global assessment and physician global assessment in rheumatoid arthritis," Journal of Rheumatology, vol. 36, no. 10, pp. 21782182, 2009.

[21] F. Gaston-Johansson, "Measurement of pain: the psychometric properties of the Pain-O-Meter, a simple, inexpensive pain assessment tool that could change health care practices," Journal of Pain and Symptom Management, vol. 12, no. 3, pp. 172-181, 1996.

[22] N. Athale, A. Sturley, S. Skoczen, A. Kavanaugh, and L. Lenert, "A web-compatible instrument for measuring self-reported disease activity in arthritis," Journal of Rheumatology, vol. 31, no. 2, pp. 223-228, 2004.

[23] N. Bellamy, J. Campbell, and J. Syrotuik, "Comparative study of self-rating pain scales in rheumatoid arthritis patients," Current Medical Research and Opinion, vol. 15, no. 2, pp. 121127, 1999.

[24] J. E. Pope, D. Khanna, D. Norrie, and J. M. Ouimet, "The minimally important difference for the health assessment questionnaire in rheumatoid arthritis clinical practice is smaller than in randomized controlled trials," Journal of Rheumatology, vol. 36, no. 2, pp. 254-259, 2009.

[25] N. Bellamy, J. Campbell, and J. Syrotuik, "Comparative study of self-rating pain scales in osteoarthritis patients," Current Medical Research and Opinion, vol. 15, no. 2, pp. 113-119, 1999.

[26] E. W. Ehrich, G. M. Davies, D. J. Watson, J. A. Bolognese, B. C. Seidenberg, and N. Bellamy, "Minimal perceptible clinical improvement with the Western Ontario and McMaster Universities Osteoarthritis Index questionnaire and global assessments in patients with osteoarthritis," Journal of Rheumatology, vol. 27, no. 11, pp. 2635-2641, 2000.

[27] K. Gottesdiener, T. Schnitzer, C. Fisher et al., "Results of a randomized, dose-ranging trial of etoricoxib in patients with osteoarthritis," Rheumatology, vol. 41, no. 9, pp. 1052-1061, 2002.

[28] M. Faucher, S. Poiraudeau, M. M. Lefevre-Colau, F. Rannou, J. Fermanian, and M. Revel, "Assessment of the test-retest reliability and construct validity of a modified Lequesne index in knee osteoarthritis," Joint Bone Spine, vol. 70, no. 6, pp. 520 $525,2003$.

[29] J. Zacher, D. Feldman, R. Gerli et al., "A comparison of the therapeutic efficacy and tolerability of etoricoxib and diclofenac in patients with osteoarthritis," Current Medical Research and Opinion, vol. 19, no. 8, pp. 725-736, 2003.
[30] F. Tubach, P. Ravaud, G. Baron et al., "Evaluation of clinically relevant changes in patient reported outcomes in knee and hip osteoarthritis: the minimal clinically important improvement," Annals of the Rheumatic Diseases, vol. 64, no. 1, pp. 2933, 2005.

[31] R. Jaeschke, J. Singer, and G. H. Guyatt, "Measurement of health status. Ascertaining the minimal clinically important difference," Controlled Clinical Trials, vol. 10, no. 4, pp. 407415, 1989.

[32] G. H. Guyatt, D. Osoba, A. W. Wu et al., "Methods to explain the clinical significance of health status measures," Mayo Clinic Proceedings, vol. 77, no. 4, pp. 371-383, 2002.

[33] H. C. W. De Vet, R. W. J. G. Ostelo, C. B. Terwee et al., "Minimally important change determined by a visual method integrating an anchor-based and a distribution-based approach," Quality of Life Research, vol. 16, no. 1, pp. 131-142, 2007. 


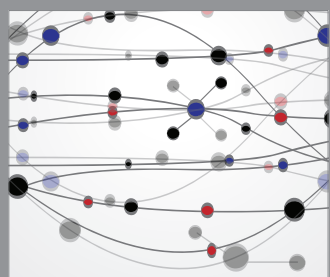

The Scientific World Journal
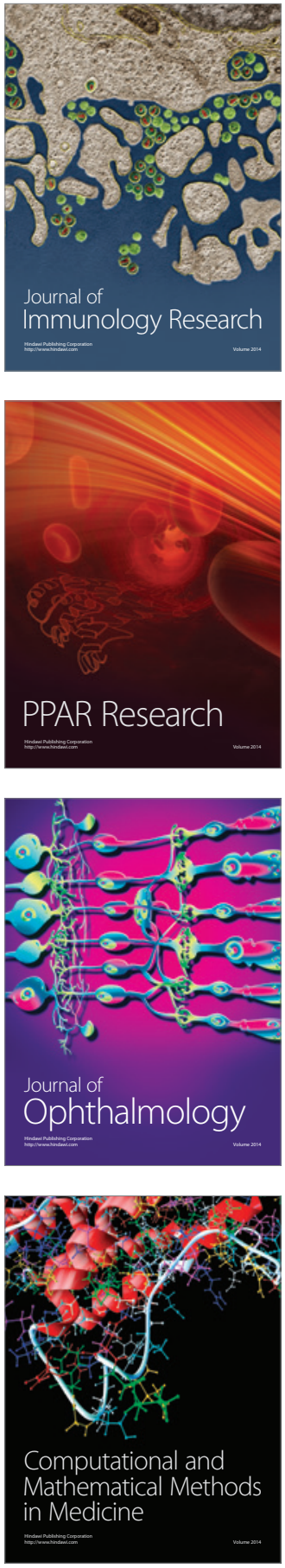

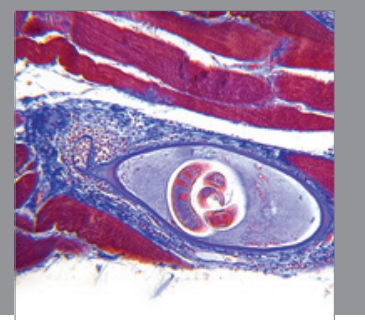

Gastroenterology

Research and Practice
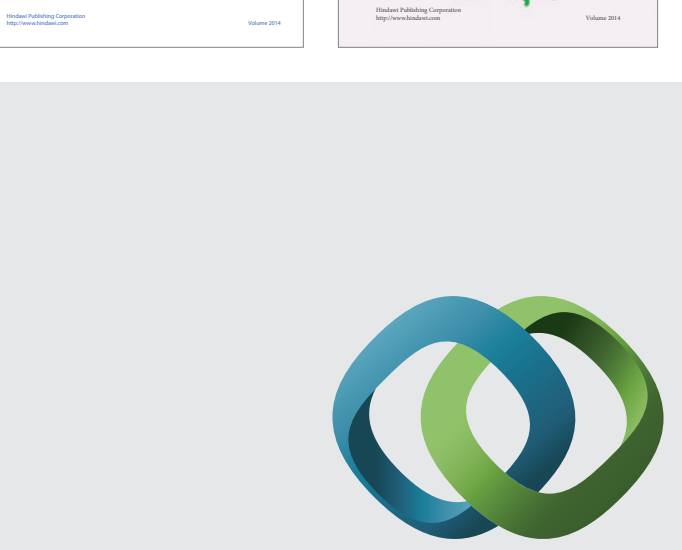

\section{Hindawi}

Submit your manuscripts at

http://www.hindawi.com
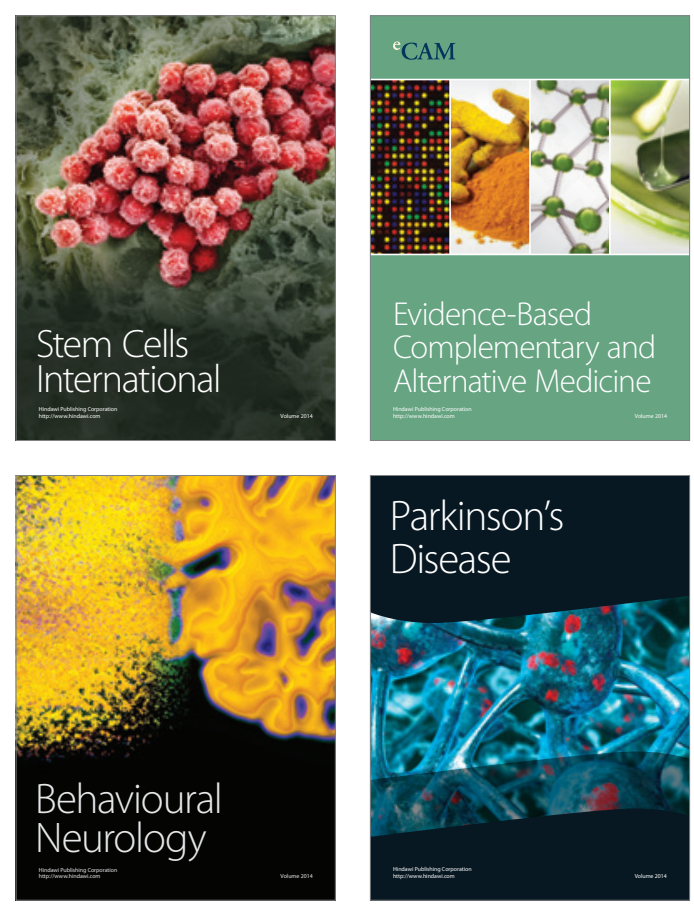

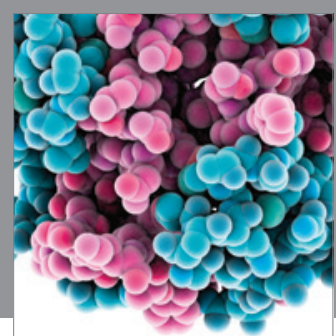

Journal of
Diabetes Research

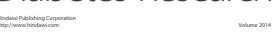

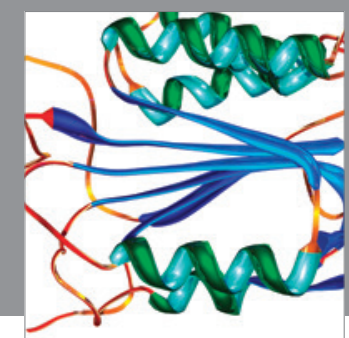

Disease Markers
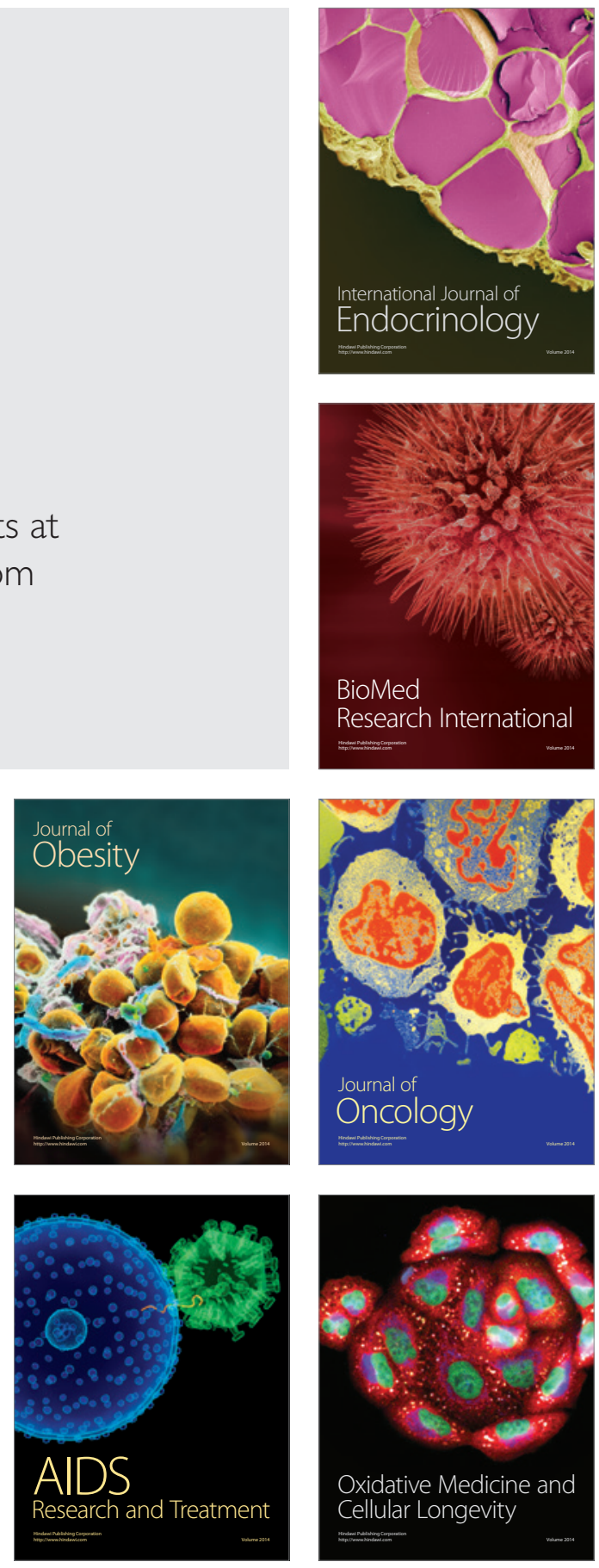